\title{
Search for WIMP dark matter produced in association with a Z boson with the ATLAS detector
}

\author{
Artem Basalaev ${ }^{1, a}$, on behalf of the ATLAS Collaboration \\ ${ }^{1}$ National Research Centre "Kurchatov Institute" B.P.Konstantinov Petersburg Nuclear Physics Institute, \\ 188300, Gatchina, Leningrad district, Russia.
}

\begin{abstract}
The search for weakly interacting dark matter particle (WIMP) candidates produced in association with a $\mathrm{Z}$ boson with the ATLAS detector at the LHC is presented. Events with large missing transverse momentum and consistent with the decay of a $\mathrm{Z}$ boson into oppositely charged electron or muon pairs were selected in analysis. Background estimates and corresponding systematic uncertainties are shown. The limits on the mass scale of the contact interaction as a function of the dark matter particle mass and the limits on the coupling and scalar particle mediator mass for $8 \mathrm{TeV}$ proton-proton collisions data are presented. Prospects for analysis using $13 \mathrm{TeV}$ proton-proton collisions data are discussed.
\end{abstract}

\section{Introduction}

Astrophysical measurements provide compelling evidence for the existence of nonbaryonic dark matter (DM) [1]. However, no experiments to date have revealed its particle nature nor proved its nongravitational interactions, if they exist [2]. At hadron colliders, weakly interacting massive particles (WIMPs), the promising candidates for dark matter particles, are predicted to be produced in pairs in some Beyond the Standard Model (BSM) theories. A WIMP would escape as undetected particle, but could be identified by a signature involving missing transverse momentum $E_{\mathrm{T}}^{\mathrm{miss}}$. Selecting events with a clear $Z$ boson signature results in a well-studied $E_{\mathrm{T}}^{\text {miss }}$ distribution. A search was performed with the ATLAS detector [3] at the LHC for deviations from $E_{\mathrm{T}}^{\text {miss }}$ distribution expected from Standard Model processes [4].

The results were examined in terms of effective field theory (EFT) models [5] and models in which the intermediate state is specified [6]. The EFTs considered in this analysis (shown on Figure 1) are expressed in terms of two parameters: mass of the DM particle $m_{\chi}$ and a mass scale $M_{*}$, which parameterizes the coupling between the WIMP and SM particles ${ }^{1}$. EFT operators D1 (scalar, spin independent), D5 (vector, spin independent), and D9 (tensor, spin dependent), following the definitions in [5], are representative of the full set of operators in which the $Z$ boson is emitted as ISR. Two examples of the dimension- $7 Z Z_{\chi \chi}$ operator mixtures are considered: one in which the $Z_{\gamma *}$ contribution is negligible and one in which it is maximal.

\footnotetext{
ae-mail: artem.basalaev@cern.ch

${ }^{1} M_{*}=\frac{M}{\sqrt{g_{\chi} g_{q}}}$, where $M$ is mediator mass, $g_{q}$ is SM-mediator coupling and $g_{\chi}$ is mediator-DM coupling.
} 
While the use of EFTs is appealing because the intermediate state mediating the parton-WIMP interaction is unknown, there are strong restrictions on EFTs validity. In particular, momentum transfer should generally be much lower than the mass scale and EFT is not a UV-complete theory. For a detailed analysis regarding validity of the EFTs for searches at the LHC please refer to [7]. To complement the EFT analysis, results were also examined in terms of a model with a scalar mediator $\eta$, with mass $m_{\eta}$, and a scalar-WIMP coupling strength $f$, shown on Figure 1(c). The production cross section is proportional to $f^{4}$. The same final state signature as predicted by the EFT, $Z_{\chi \bar{\chi}}$, is produced.

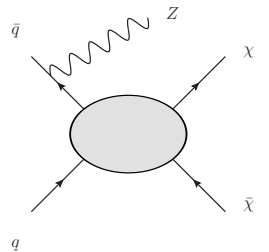

(a)

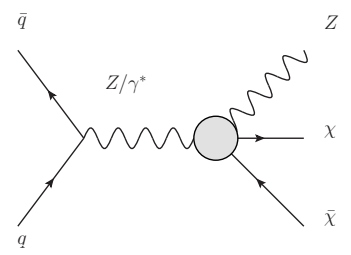

(b)
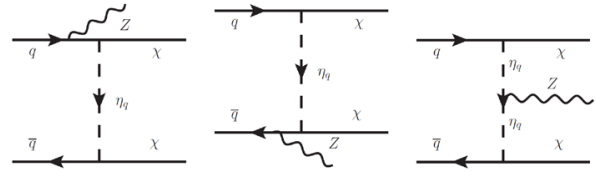

(c)

Figure 1. Diagrams are shown for EFTs with an ISR operator (a), with a $Z Z_{\chi \bar{\chi}}$ operator (b), and for models with specified mediator (c).

\section{Event Selection and Background Estimation}

Electron candidates are reconstructed from isolated energy deposits in the electromagnetic calorimeter with a shower shape consistent with electrons or photons, matched to inner detector tracks [8]. Muon candidates are reconstructed combining tracks independently found in the muon spectrometer and inner tracking detector [9]. Jets are reconstructed using the anti- $k_{t}$ algorithm [10] with a radius parameter $R=0.4$. Basic kinematic cuts are also applied, along with some additional selection to ensure good separation between reconstructed objects [4].

The invariant mass of a lepton pair is required to be consistent with that of a $Z$ boson, $76 \mathrm{GeV} \leq$ $\mathrm{M}_{\ell \ell} \leq 106 \mathrm{GeV}$. Events are required to pass event quality checks for errors in sub-detectors during recording, and at least one of the trigger requirements: single-lepton with higher $p_{\mathrm{T}}$, or lepton-pair with lower $p_{\mathrm{T}}$. The $E_{\mathrm{T}}^{\text {miss }}$ is expected to be back-to-back with the $Z$ boson. This is accounted for in the $\Delta \phi\left(Z, E_{\mathrm{T}}^{\mathrm{miss}}\right)$ variable, which is required to be greater than 2.5 . The absolute value of the pseudorapidity of the dilepton system, $\left|\eta_{l l}\right|$, must be less than 2.5 . Any possible imbalance, implying the presence of fake $E_{\mathrm{T}}^{\mathrm{miss}}$, is accounted for by an upper threshold on the fractional $p_{\mathrm{T}}$ difference variable, defined as $\left|E_{\mathrm{T}}^{\mathrm{miss}}-p_{T}^{\ell \ell}\right| / p_{T}^{\ell \ell}<0.5$. Events are removed if they contain jets with $p_{\mathrm{T}}>25 \mathrm{GeV}$ to suppress top-quark pair background. Events containing a third lepton with $p_{\mathrm{T}}>7 \mathrm{GeV}$, satisfying looser identification requirements are removed to suppress diboson background. The dark matter models considered here have different $E_{\mathrm{T}}^{\text {miss }}$ spectra, leading to a variety of optimal lower thresholds of $E_{\mathrm{T}}^{\mathrm{miss}}$. Four inclusive signal regions are defined with lower thresholds in $E_{\mathrm{T}}^{\text {miss }}$ of $150,250,350$, and $450 \mathrm{GeV}$.

The dominant background process is $Z Z \rightarrow \ell^{+} \ell^{-} v \bar{v}$, an irreducible background. Reducible backgrounds may have jets produced in association with two leptons, where the jets are misidentified or unreconstructed, such as $t \bar{t} \rightarrow \ell^{+} v b \ell^{-} \bar{v} \bar{b}, Z Z \rightarrow \ell^{+} \ell^{-} q \bar{q}, W Z \rightarrow \bar{q} q^{\prime} \ell^{+} \ell^{-}$or $Z+$ jets (including decays to $\tau$ leptons). The other important background is $W W \rightarrow \ell^{+} v \ell^{-} \bar{v}$, which may only be reduced through the mass window requirement. Additional reducible sources may produce events with more 
than two leptons where the additional leptons are misidentified or not reconstructed, or less than two leptons where jets are misidentified as leptons, such as $W Z \rightarrow \ell v \ell^{+} \ell^{-}$and $W+$ jets, respectively.

Data-driven techniques for background estimation were used where possible. Otherwise, MC simulation samples were used, obtained with MC generators such as SHERPA, PowHEg. Detailed information on background estimations is provided in [4].

The $Z Z$ and $W Z$ backgrounds are estimated from MC simulations. The $W W, t \bar{t}, W t$, and $Z \rightarrow t \bar{t}$ backgrounds are estimated from data using the absence of signal in the $e \mu$ channel and the relative production rate of $1: 1: 2$ for the ee, $\mu \mu$, and $e \mu$ channels. An $e \mu$ control region similar to the signal region is defined, and the background estimate for the ee and $\mu \mu$ signal regions is obtained from the number of $e \mu$ events in the control region after correcting for different acceptances and efficiencies. The $Z+$ jets background is estimated using two data-driven techniques: $A B C D$ method [11] using uncorrelated variables $E_{\mathrm{T}}^{\mathrm{miss}}$ and $\eta_{l l}$, and by fitting the distributions of $\Delta \phi\left(Z, E_{\mathrm{T}}^{\mathrm{miss}}\right)$ and $E_{\mathrm{T}}^{\mathrm{miss}}$ at small values and extrapolating them to the signal regions. The $W+$ jets background is estimated by reversing the electron isolation condition and loosening identification requirements for one electron in order to obtain a data sample enriched in jets reconstructed as electrons.

\section{Results and Conclusions}

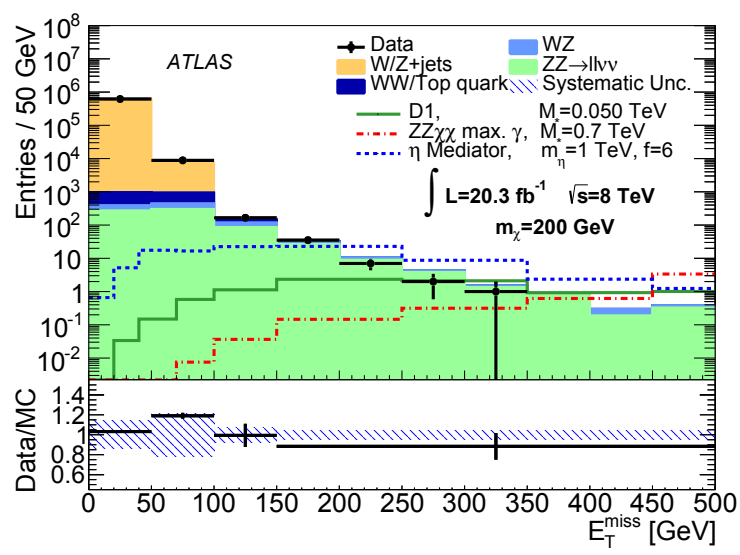

Figure 2. $E_{\mathrm{T}}^{\text {miss }}$ distribution is presented after all event selections other than the $E_{\mathrm{T}}^{\text {miss }}$ thresholds for the observed data [4].

The search was performed on $20.3 \mathrm{fb}^{-1}$ of ATLAS dataset collected in 2012 at $\sqrt{s}=8 \mathrm{TeV}$. Theoretical and experimental systematic uncertainties are estimated for all studied backgrounds, including uncertainties associated with pileup reweighting, luminosity calculation, object and $E_{\mathrm{T}}^{\text {miss }}$ reconstruction algoritms and methods of background estimation. Data yields with associated statistical and systematic uncertainties are shown on Table 1 . The $E_{\mathrm{T}}^{\text {miss }}$ distribution after applying all selection requirements other than the $E_{\mathrm{T}}^{\text {miss }}$ thresholds for the observed data, the expected SM backgrounds, and the hypothetical $p p \rightarrow Z_{\chi \bar{\chi}}$ signals for various values of the mass scale are shown on Figure 2. No significant deviations from the SM predictions have been observed. 
Table 1. Observed yields and expected SM backgrounds in each signal region. Statistical, systematic, and luminosity uncertainties are combined [4].

\begin{tabular}{|lcccc|}
\hline & \multicolumn{4}{c|}{$E_{\mathrm{T}}^{\text {miss }}$ Threshold, GeV } \\
Process & 150 & 250 & 350 & 450 \\
$Z Z$ & $41 \pm 15$ & $6.4 \pm 2.4$ & $1.3 \pm 0.5$ & $0.3 \pm 0.1$ \\
$W Z$ & $8.0 \pm 3.1$ & $0.8 \pm 0.4$ & $0.2 \pm 0.1$ & $0.1 \pm 0.1$ \\
$W W, t \bar{t}$, & $1.9 \pm 1.4$ & $0_{-0.0}^{+0.7}$ & $0_{-0.0}^{+0.7}$ & $0_{-0.0}^{+0.7}$ \\
$Z \rightarrow \tau^{+} \tau^{-}$ & & & & \\
$Z+$ jets & $0.1 \pm 0.1$ & $\ldots$ & $\ldots$ & $\ldots$ \\
$W+$ jets & $0.5 \pm 0.3$ & $\ldots$ & $\ldots$ & $\ldots$ \\
\hline Total & $52 \pm 18$ & $7.2 \pm 2.8$ & $1.4 \pm 0.9$ & $0.4_{-0.4}^{+0.7}$ \\
Data & $\mathbf{4 5}$ & $\mathbf{3}$ & $\mathbf{0}$ & $\mathbf{0}^{-0.7}$ \\
\hline
\end{tabular}

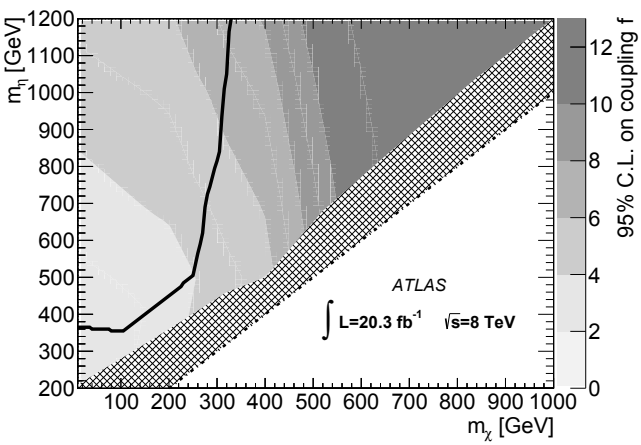

(a)

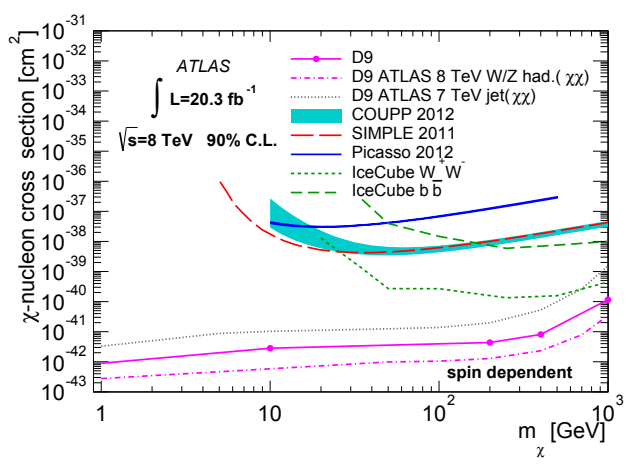

(c)

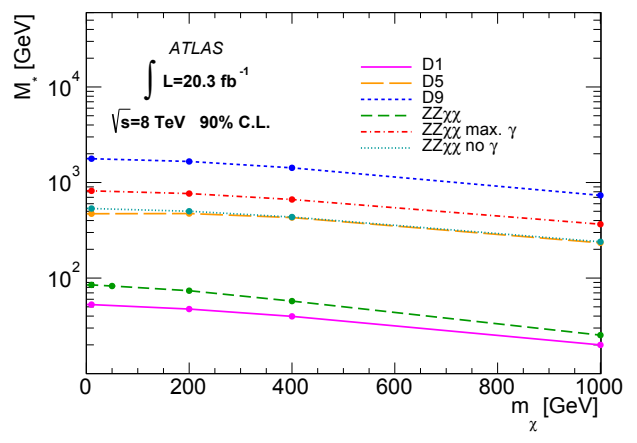

(b)

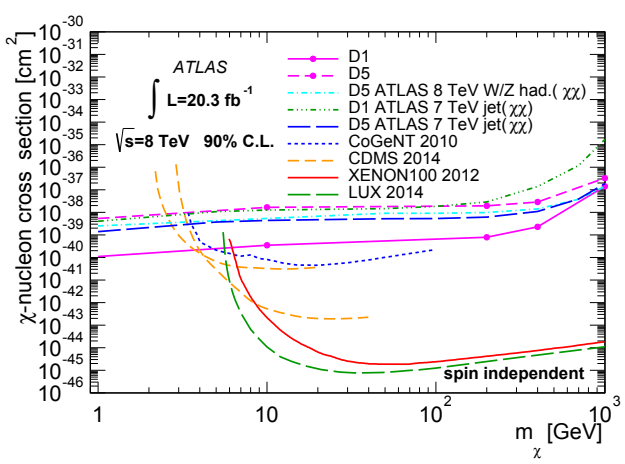

(d)

Figure 3. Observed upper limits at $95 \% \mathrm{CL}$ are shown on the coupling constant $f$ of the scalar-mediator theory as a function of mediator mass $m_{\eta}$ and dark matter particle mass $m_{\chi}$ [4] (a). Observed 90\% C.L. lower limits are shown on the mass scale $M_{*}$ of considered effective field theories as a function of $m_{\chi}$ [4] (b) and on the $\chi$ nucleon scattering cross section as a function of $m_{\chi}$ for the spin-dependent (c) and spin-independent (d) effective operators [4].

The $95 \%$ C.L. upper limits on the on the coupling constant $f$ of the scalar-mediator theory and the $90 \%$ C.L. lower limits on the mass scale $M_{*}$ of the effective field theory, which parameterizes 
the coupling between the WIMP and SM particles, are presented on Figure 3(a) and Figure 3(b) correspondingly.

Additionally the limits are translated into $\chi$-nucleon scattering cross section and compared with several other ATLAS searches and direct searches for spin-depentent (Figure 3(c)) and spinindependent (Figure 3(d)) cases.

\subsection{Prospects in Run 2}

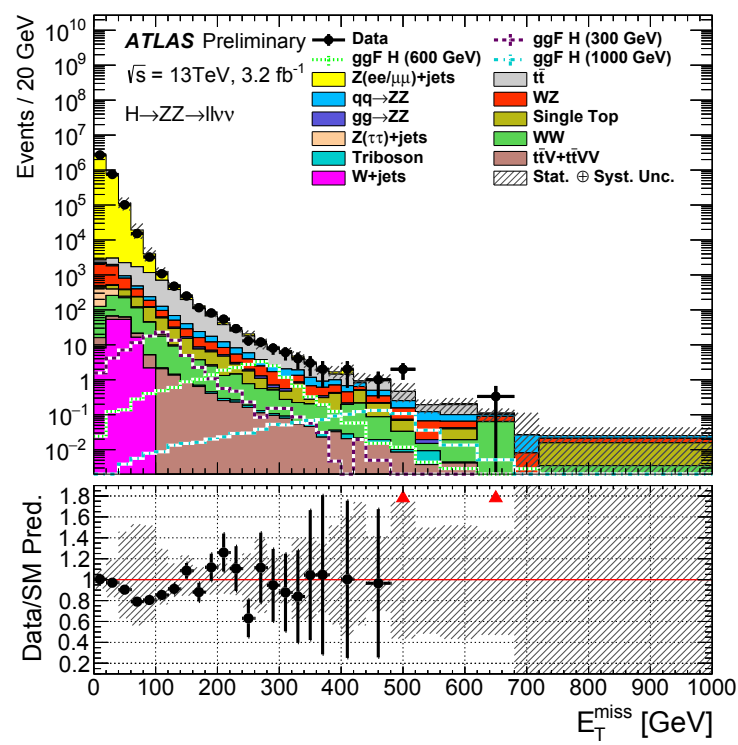

Figure 4. $E_{\mathrm{T}}^{\text {miss }}$ distribution is shown after the dilepton mass requirement for the search for additional heavy Higgs boson in $\ell \ell v v$ final state, performed in Run 2 on 2015 data [12].

Run 2 of the LHC is steadily ongoing, with proton-proton collision energy reaching unprecedented $\sqrt{s}=13 \mathrm{TeV}$. The integrated luminosity collected in Run 1 has already been surpassed, promising increase in sensitivity for many analyses, including the search discussed here. Presented on Figure 4 are the background estimates with 2015 data for the search for additional heavy Higgs boson, which is utilizing the same $\ell \ell v v$ final state and the same processes are considered as background.

\section{References}

[1] E. Komatsu et al., Astrophys. J. Suppl. Ser. 192, 18 (2011)

[2] K.A. Olive et al. (Particle Data Group), Chin. Phys. C38, 090001 (2014)

[3] ATLAS Collaboration, JINST 3, S08003 (2008)

[4] ATLAS Collaboration, Phys. Rev. D 90, 012004 (2014)

[5] J. Goodman, M. Ibe, A. Rajaraman, W. Shepherd, T.M.P. Tait, H.B. Yu, Phys. Rev. D 82, $116010(2010)$

[6] N. Bell, J. Dent, A. Galea, T. Jacques, L. Krauss, T. Weiler, Phys. Rev. D 86, 096011 (2012) 
[7] D. Abercrombie et al., ArXiv e-prints (2015), 1507.00966

[8] ATLAS Collaboration, Eur. Phys. J. C 72, 1909 (2012)

[9] ATLAS Collaboration, J. High Energy Phys. 12, 060 (2010)

[10] M. Cacciari, G.P. Salam, G. Soyez, J. High Energy Phys. 04, 063 (2008)

[11] ATLAS Collaboration, Phys. Lett. B 719, 299 (2013)

[12] ATLAS Collaboration, Tech. Rep. ATLAS-CONF-2016-012 (2016), https://cds. cern.ch/record/2140833 